\title{
REFLEXIONES PARA UNA BIOÉTICA IMPLICADA EN LA CULTURA DE LOS DERECHOS HUMANOS
}

\author{
Silvia Brussino ${ }^{1, a}$
}

\begin{abstract}
RESUMEN
La Bioética puede entenderse como una reflexión crítica sobre la vida y la salud, como una nueva forma de tomar decisiones en esos ámbitos, como un movimiento de reforma social y también como una disciplina académica. En cualquiera de los casos, la Bioética implica un diálogo interdisciplinario e intercultural. A su vez, los derechos humanos, como pautas morales universales, proporcionan una base plausible para el diálogo intercultural, permitiendo identificar un núcleo de valores transculturales que funcionen como "mínimos morales" en las arenas del diálogo entre culturas diversas y la búsqueda de consensos internacionales. En este trabajo se plantea la triple vinculación (histórico-genealógica, conceptual y práctica) de la bioética y los derechos humanos y se reflexiona sobre algunas condiciones que deberían tomarse en cuenta para una bioética implicada en la cultura de los derechos humanos.
\end{abstract}

Palabras clave: Bioética; Derechos humanos; Ética (fuente: DeCS BIREME).

\section{REFLECTIONS FOR HAVING BIOETHICS INVOLVED IN THE HUMAN RIGHTS CULTURE}

\section{ABSTRACT}

\begin{abstract}
Bioethics can be interpreted as a critical reflection on life and health, a new way to make decisions in these fields, a social reform movement, and an academic discipline. In any case, Bioethics implies an interdisciplinary and intercultural dialogue. At the same time, human rights, as universal moral guidelines, provide a plausible basis for this intercultural dialogue, for they enable the identification of a core of transcultural values that can work as "moral minima" in the dialogue among different cultures and the search for international consensuses. This article sets forth the triple connection (historical-genealogical, conceptual and practical) between bioethics and human rights, and reflects on some of the conditions that should be taken into account for bioethics to be involved in the human rights culture.
\end{abstract}

Key words: Bioethics; Human rights; Ethics (source: MeSH NLM).

\section{SOBRE EL SIGNIFICADO \\ DE UNA "CULTURA DE LOS DERECHOS HUMANOS"}

Si consideramos la polisemia del término "cultura" y su carga histórica, conviene despejar lo que entendemos por "cultura de los derechos humanos". Sin entrar en los detalles de la génesis del término, una primera referencia que evoca es la antítesis cultura-naturaleza del siglo XVIII, una vez que quedó fijado su campo semántico en relación al género humano, es decir, una vez que se hubo desplazado el objeto de la acción de cultivar desde la tierra al espíritu humano, ligándolo así al desarrollo de las facultades humanas por obra de la educación, en franca oposición al estado de naturaleza de un espíritu no cultivado. Desde esta vertiente, consagrada por el Diccionario de la Academia Francesa en su edición de 1798, la palabra cultura se asocia a la idea de progreso, de evolución, de saberes acumulados y transmitidos por la humanidad -como totalidad no diferenciada- en el curso de la historia, entendida esta como proceso universal y progresivo. De allí que en la ideología de

\footnotetext{
Universidad Nacional del Litoral. Santa Fe, Argentina.

a Filósofa magíster en Bioética

Recibido: 19-06-12 Aprobado: 14-11-12
}

Citar como: Brussino S. Reflexiones para una bioética implicada en la cultura de los derechos humanos. Rev Peru Med Exp Salud Publica. 2012;29(4):561-65. 
las Luces, la palabra cultura se asoció a "civilización", como ese megaproceso en el que estaba embarcada toda la humanidad, incluyendo a los denominados pueblos "salvajes", ya que este proceso les permitiría salir de la ignorancia y la irracionalidad mediante el mejoramiento de las instituciones políticas, la legislación y la educación.

Una segunda referencia evocada por el término "cultura" es justamente la oposición al término "civilización", surgida en el seno de la intelectualidad alemana del siglo XVIII e identificada con el espíritu del pueblo (volksgeist). Este significado se fue acentuando durante el siglo XIX con el romanticismo alemán, designando una particular comunidad cultural de origen que precede y reclama la unidad política, por lo que esta idea de cultura será el basamento del Estado-nación alemán.

Podemos decir entonces que en el origen del término "cultura" coexisten un elemento universalista, que remite a la unidad del género humano; y un elemento particularista, como comunidad étnico-racial previo a toda configuración política.

Ambos sentidos del término "cultura" se encuentran hoy implicados de manera muy compleja, constituyendo la cuestión central del multiculturalismo. No se trata solamente de la oposición civilización universal/cultura nacional, como vimos, sino del enfrentamiento político de los grupos culturalmente diversos dentro de una misma nación.

Hacer referencia al multiculturalismo no es solamente señalar la coexistencia de la diversidad cultural dentro de una sociedad sino que es también "reivindicar un reconocimiento político oficial de la pluralidad cultural y un tratamiento público equitativo de todas las colectividades culturales" (1). Esto implica acciones por parte de los estados que deben incorporar las diferencias culturales en sus constituciones, legislaciones e instituciones, formulando además "políticas que aseguren que los intereses de grupos particulares (se trate de minorías o mayorías históricamente marginadas) no sean ignorados ni invalidados por la mayoría o por otros grupos dominantes" (2).

La cultura de los derechos humanos parece instalarse (al menos en su pretensión de validez) en el "ámbito universalista" que corresponde a toda la especie humana. Pero también pertenece a la cultura de los derechos humanos el "reconocimiento de la diversidad cultural", proclamada como un derecho humano fundamental por la Declaración Universal de Derechos Humanos, en su artículo 27 y como "patrimonio común de la humanidad", cuya defensa se erige como "imperativo ético indisociable del respeto de la dignidad de la persona" (3).

Una cultura, a su vez, comprende elementos materiales y elementos ideales, ambos configuran su realidad: los primeros son hechos constatables, los segundos se expresan más bien en un sentido heurístico, como una dirección de búsqueda colectiva. Hay una tensión entre los elementos materiales y los ideales de una cultura, pero es una tensión necesaria que constituye su propia dialéctica. Buenos ejemplos de esta tensión son las relaciones entre el derecho y la ética o entre la política y la ética: son dimensiones diferentes, pero se requieren mutuamente. La ética expresa ideales que necesitan plasmarse jurídica y políticamente para ganar fuerza y concreción, pero un sistema jurídico o político ha de sostenerse necesariamente en fundamentos éticos para ganar legitimidad y sustentabilidad.

Una comprensión disociada de esta tensión dialéctica conduce a una visión distorsionada de los acontecimientos históricos y tiende a cristalizar la cultura en un punto fijo privándola de su propia dinámica, que se nutre de la tensión entre los hechos y los ideales iniciados en esos mismos hechos. Sin esa tensión no se entiende la posibilidad de cambios en los procesos históricos.

La cultura de los derechos humanos contiene también una tensión entre dos maneras de entender el "discurso" de los derechos humanos. En efecto, hay una interpretación negativa de este discurso como paternalista, etnocéntrico o mera retórica de las naciones dominantes para suavizar los efectos devastadores del poderío que ejercen en el escenario internacional. La otra interpretación del término "discurso" aplicado a los derechos humanos se asimila a la propuesta de una ética discursiva o dialógica tal como la han desarrollado autores como Habermas y Apel, y significa en principio:

- El reconocimiento de la igualdad de todos los seres humanos en su condición de tales;

- Una apuesta por un modo de racionalidad incluyente de una diversidad que es polifónica, que se expresa en distintas voces;

- La posibilidad de una búsqueda colaborativa de la verdad, de la justicia y de la paz entre seres humanos que son culturalmente diversos, donde la única manera de resolver nuestros conflictos es a través de la comprensión de la posición del otro y del respeto de esa posición en la búsqueda de convergencias;

- Una forma de interacción humana basada en el entendimiento mutuo a través del diálogo y el compromiso de exclusión de la violencia; 
- La revisión crítica de los propios supuestos de la cultura occidental donde vieron la luz los derechos humanos. No hay pacificación y no hay posibilidades de diálogo si no se acomete en serio la reflexión crítica de estos supuestos, que me parece están en la base de todo el colonialismo y de las nuevas formas de colonialidad;

- Un modo de entender el poder como construcción vinculante y compartida, no como dominio de una parte sobre la otra. Esto implica un compromiso político con una forma de democracia deliberativa, no meramente formal sino participativa;

- Un modo de tomar en serio los derechos humanos, como compromiso solidario con todos y cada uno de los seres humanos que se encuentran en situación de vulnerabilidad en cualquier lugar del mundo.

La vinculación entre bioética y derechos humanos, se apoya en este sentido del discurso de los derechos humanos, sin desmedro de la justificación empírica que pueda hacerse de la interpretación negativa que he señalado.

\section{VINCULACIÓN DE LA BIOÉTICA Y LOS DERECHOS HUMANOS}

Considerando algunas caracterizaciones que están bastante consensuadas, la Bioética puede entenderse como una reflexión crítica sobre la vida y la salud, como una nueva forma de tomar decisiones en los diferentes ámbitos que atañen a la vida y la salud, como un movimiento de reforma social y también como una disciplina académica. En cualquiera de los casos Bioética implica diálogo interdisciplinario e intercultural. Interdisciplinario en el sentido de la complejidad propia de los fenómenos de la vida y la salud; la no neutralidad de los discursos disciplinarios en la medida en que no existen "hechos puros" que nos presente ni la Biología, ni la Física, ni las Ciencias Sociales.

Diálogo intercultural en el sentido de que los valores dependen en gran medida de los contextos culturales por lo cual vale la pena plantearse si existen algunos valores universales o los valores son únicamente particulares, dependiendo exclusivamente de un contexto histórico-cultural.

También el campo/objeto de estudio de la Bioética presenta controversias según sus distintas versiones, pudiendo distinguirse:

- Una Bioética principialista, que responde a la perspectiva de los países centrales, básicamente articulada en torno a la primacía de la autonomía como atributo principal del individuo.
- Una Bioética personalista, más bien ligada a la cosmovisión cristiana, centrada en el valor absoluto de la vida humana.

\section{NUEVOS ENFOQUES SOCIALES DE LA BIOÉTICA}

Si bien la Bioética en su versión más difundida, la angloamericana, surgió como reflexión sobre situaciones emergentes del desarrollo científicotecnológico aplicado a la salud humana, vistas las cosas desde los países periféricos, no se tardó en advertir que la Bioética tiene su campo de reflexión y actuación en situaciones persistentes ${ }^{(4)}$, tales como la pobreza extrema, el analfabetismo, la exclusión social y la injusticia estructural ${ }^{(5,6)}$, que afectan a millones de personas en nuestros países.

Es desde esta perspectiva social de la Bioética que ha ido cobrando cada vez mayor fuerza y consenso la vinculación de la Bioética con los derechos humanos, que dista de ser una construcción acabada y homogénea, reconociéndose más bien como un "conjunto de concepciones [de la Bioética] orientadas a las obligaciones universales como punto de partida, los fines, la verdad, la justicia y la memoria histórica como supuesto de construcción de la moral" (7).

Avalando esta perspectiva, existe a mi modo de ver una triple vinculación entre la Bioética y los derechos humanos:

- Una vinculación histórico-genealógica, en tanto que se identifican como antecedentes importantes de la Bioética al Código de Nüremberg (1947) y a la Declaración de Helsinki (1964), documentos que comparten con la Declaración Universal de los Derechos Humanos (1948), los principios de una moral universalista basada en la igual dignidad de los seres humanos en su carácter de tales.

- Una vinculación conceptual, articulada en torno a la centralidad del concepto de dignidad tanto para la teoría y la práctica de los derechos humanos como de la Bioética.

- Una vinculación práctica, que tiene su base en el sistema internacional de derechos humanos plasmada en pactos, convenciones y declaraciones como las de la UNESCO sobre el Genoma Humano y los Derechos Humanos (1997), sobre los Datos Genéticos Humanos (2003) y sobre Bioética y Derechos Humanos (2005). 


\section{CULTURA DE LOS DERECHOS HUMANOS Y DIÁLOGO INTERCULTURAL}

Los derechos humanos como pautas morales universales, proporcionan una base plausible para el diálogo intercultural. Sin embargo, es necesario plantear las condiciones de este diálogo, habida cuenta de que se lleva a cabo en el mundo tal cual está, es decir, en un mundo atravesado por situaciones de grandes asimetrías que tienden a consolidarse y profundizarse fácticamente por el uso irracional del poder, ya sea éste económico, científico-técnico, político o militar.

Como se sabe, gran parte de esas asimetrías han sido generadas por la modernidad europea en el mismo clima cultural que los derechos humanos, lo cual tiñe de sospecha toda su producción planteando un panorama sombrío para una ética global. Como observa Stolkiner: El constructo 'derechos' humanos entraña, en sí, en su origen y en su potencialidad, una paradoja que permite situarlo tanto del lado de las políticas transformadoras como de las de dominación ${ }^{(8)}$.

No obstante, y aun asumiendo las dificultades teóricas que presenta una adecuada definición de la dignidad humana ${ }^{(9)}$ es importante la afirmación práctica de esta idea, dotada de fuerte poder intuitivo, que nos provee de un sentido de igualdad entre los seres humanos, como punto de partida de toda valoración ulterior: solo sobre la base del reconocimiento no banalizado de la igualdad como elemento central de una cultura de los derechos humanos se pueden discutir los componentes de la diversidad cultural que merecen ser fomentados y cuáles son también los límites de ese derecho ${ }^{(3,10)}$.

El hecho de acercarnos a culturas diferentes no significa que tengamos que "convertirnos" a esa cultura: querer comprender no significa que estemos ya de acuerdo. Hablar de transculturalidad en el diálogo tiene como condición que podamos identificar un núcleo de valores transculturales que funcionen como "mínimos morales" en las arenas del diálogo intercultural y la búsqueda de consensos internacionales.

Pienso que realizar una suerte de apología de las culturas y de la diversidad cultural por la diversidad cultural misma, como lo hacen algunas posturas relativistas en su oposición al universalismo moral, nos conduce a un error práctico grave, porque convierte en indiferentes todas las diferencias, en la medida en que hace inútil cualquier discusión ${ }^{(11)}$, privándonos de un criterio para discernir qué prácticas no son buenas para ningún ser humano, como por ejemplo la tortura o la explotación, cualquiera sea el contexto cultural en el que se produzcan.
Por otro lado, es importante no plantear los derechos humanos desde una suerte de neutralidad respecto de los contenidos valorativos de las culturas. En cualquier asunto complejo de importancia humana no hay un "ojo inocente", ninguna forma de ver al mundo que sea enteramente neutral y esté libre de conformaciones culturales. Buena parte de la filosofía del siglo $X X$ se ha dedicado a poner en evidencia que no hay contenido de la conciencia que no esté "contaminado" por alguna interpretación. Pero de ello no se deriva que todas las interpretaciones del mundo sean igualmente válidas, como pretende el relativismo, de la misma manera que tampoco nos obliga a renunciar de antemano a la postulación de unos valores morales universales contenidos en los derechos humanos. Como observa Martha Nussbaum acertadamente: Transformando cada tradición en la última palabra, nos privamos a nosotros mismos de toda otra norma más general de tolerancia o respeto que pueda ayudarnos a limitar la intolerancia de las culturas ${ }^{(12)}$.

Considero que los derechos humanos no pueden ser impuestos sino que deben ser puestos a prueba en el diálogo intercultural. Decir "puestos a prueba", indica que la pretensión de validez universal del contenido de estos derechos tiene que medirse en el terreno del diálogo crítico con otras culturas y confrontarse con otros ethos de culturas diferentes ${ }^{(13)}$. Pero al mismo tiempo, desde la convicción de que los derechos humanos tienen algo interesante para proponer a todas las culturas, a todos los seres humanos en su condición de tales. Por eso son candidatos plausibles a la universalización, que no es lo mismo que una universalidad lograda de antemano y cristalizada de una vez y para siempre, sino que se tiene que ir ganando con trabajo, en el conflicto de las diversas convicciones ${ }^{(11)}$.

\section{REFLEXIONES FINALES}

Es importante tener presente que los derechos humanos son producto de un proceso histórico, que han sido acuñados en muchas luchas por el reconocimiento mutuo de los seres humanos, que ese proceso no ha sido meramente discursivo, como tampoco es lineal y clausurado. Los procesos históricos tienen avances $y$ retrocesos $y$, aunque hoy tengamos declaraciones y un sistema internacional de derechos humanos, hay marchas y contramarchas. Es por eso que no se puede "bajar la guardia": estamos imbuidos en procesos históricos y tenemos que lidiar con ese rasgo de la historicidad que es su fragilidad. Nada es una adquisición definitiva en materia humana. Son las luchas por ese reconocimiento -constituyente de identidad- del que nadie puede sustraerse, las que atraviesan la historia 
de los derechos humanos y la dotan de su particular carácter reivindicativo. Es precisamente la brecha entre lo que pretendemos y lo que los demás están dispuestos a concedernos lo que constituye el drama del reconocimiento, que se juega en el espacio público internacional y que determina un movimiento incesante en las sucesivas generaciones de derechos humanos. Debemos pensar que esta es una tarea inacabable, al menos mientras la historia continúe en marcha y el futuro permanezca abierto. Esto sugiere que no pueden cristalizarse los logros en materia de derechos humanos -que no son pocos- ya que siempre habrá aspectos de la diversidad de modos de vida y de interacción humana que estarán pugnando por ser reconocidos en el espacio público; todo hace pensar que la misma dinámica de las sociedades generará nuevos conflictos o una nueva configuración de los conflictos ya existentes.

Así como se impone "relativizar el relativismo cultural" rechazando la idea de neutralidad en el diálogo intercultural, tanto el discurso como la práctica de los derechos humanos deben ser sometidos a una autocrítica permanente. Muchas de las decepciones actuales sobre la potencialidad normativa transcultural de estos derechos, se deben a una cierta ceguera en las políticas de desarrollo y de gestión de las diferencias culturales: la falta de una refinada percepción de los múltiples y complejos elementos contextuales en los que se juegan los conflictos del reconocimiento, conduce al fracaso de muchos bienintencionados proyectos de intervención para mejorar la calidad de vida de las personas. Por eso, requieren un cuidadoso examen de los elementos contextuales y las condiciones materiales que impiden el ejercicio concreto de las libertades a personas o grupos, aunque estas figuren entre las garantías constitucionales o las leyes de un país. Sobran ejemplos en el mundo entero sobre la inutilidad de unos derechos escritos que se convierten en letra muerta cuando no están dadas las condiciones económicas, sociales, políticas y educativas para que las personas puedan gozar efectivamente de los mismos. No obstante, buena parte del trabajo en materia de derechos humanos ha permitido identificar y remover diferentes tipos de obstáculos que impiden que las personas puedan gozar de estos derechos. Existen razones para pensar que su ampliación a todas las personas y todos los pueblos de la tierra será la gran empresa moral y política del siglo XXI.

Fuentes de financiamiento: este trabajo se enmarca en el Proyecto Especial (PE) de Investigación y Desarrollo (CAI+D) "Etica profesional y conocimiento en ciencias de la salud" desarrollado entre 2009 y 2012, financiado por la Universidad Nacional del Litoral (Argentina).

Conflictos de interés: el autor declara no tener ningún conflicto de interés.

\section{REFERENCIAS BIBLIOGRÁFICAS}

1. Cuche D. La noción de cultura en las ciencias sociales. Buenos Aires: Nueva Visión; 2002.

2. Programa de las Naciones Unidas para el Desarrollo (PNUD). Informe sobre desarrollo humano 2004. Madrid: Mundi-Prensa; 2004.

3. UNESCO. Declaración Universal sobre la diversidad cultural, adoptada por la $31^{\circ}$ Asamblea General el 3 de noviembre de 2001. París: UNESCO; 2002

4. Garrafa V. Dimensão da ética em saúde pública. São Paulo: Faculdade de Saúde Pública/USP; 1995.

5. Vidal S. Iniquidad y Desarrollo Humano - una mirada desde la bioética. O Mundo da Saúde 2004;28(3):304-14.

6. Red Bioética, UNESCO. Carta de Buenos Aires [Internet]. Buenos Aires: UNESCO, REDBIOÉTICA; 2004 [citado el 12 de julio del 2011]. Dis- ponible en: http://www.unesco.org. uy/shs/red-bioetica/fileadmin/shs/redbioetica/CartaBuenosAires-RED.pdf

7. Tealdi JC. Bioética de los derechos humanos en Diccionario Latinoamericano de Bioética. Bogotá: UNESCO/Universidad Nac. de Colombia; 2008.

8. Stolkinner A. Derechos humanos y derecho a la salud en América Latina: la doble faz de una idea potente. Rev Medicina Social. 2010;5(1):89-95.

9. Bohórquez Monsalve V, Aguirre Román J. Las tensiones de la dignidad humana: conceptualización y aplicación en el derecho internacional de los derechos humanos. Sur Rev Int Direitos Human. 2009;6(1):41-63.

10. UNESCO. Declaración Universal sobre Bioética y Derechos Humanos. Asamblea General el 31 de octubre de
2005. Revista Selecciones de Bioética. 2006;(10):6-16.

11. Ricoeur P. Sí mismo como otro. Madrid: Siglo XXI; 1996.

12. Nussbaum M. Las mujeres y el desarrollo humano. Barcelona: Herder; 2002.

13. De Zan J. La ética, los derechos y la justicia. Montevideo. Fundación Konrad Adenauer Uruguay; 2004.

Correspondencia: Silvia Liliana Brussino Dirección: Pedro Centeno 1912 -3000Santa Fe, Argentina.

Teléfono:(54 03) 424559564

Correoelectrónico:sbrussino@hotmail.com 